DIVISION OF THE HUMANITIES AND SOCIAL SCIENCES

CALIFORNIA INSTITUTE OF TECHNOLOGY

PASADENA, CALIFORNIA 91125

STATISTICAL ANALYSIS OF THE ADDITIVE AND MULTIPLICATIVE HYPOTHESES OF MULTIPLE EXPOSURE SYNERGY FOR COHORT AND CASE-CONTROL STUDIES

Jeffrey A. Dubin

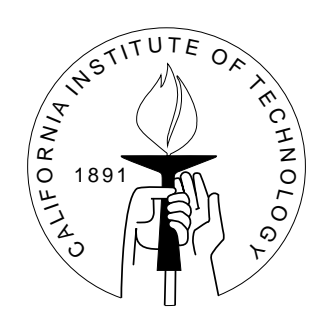

SOCIAL SCIENCE WORKING PAPER 1066 


\title{
Statistical Analysis of the Additive and Multiplicative Hypotheses of Multiple Exposure Synergy for Cohort and Case-Control Studies
}

\author{
Jeffrey A. Dubin
}

\begin{abstract}
This paper considers hypotheses tests for synergistic relationships in epidemiological studies. Two hypotheses are considered. First, I develop tests of the additive hypothesis which states that the combined risk from two sources of exposure is the sum of each risk taken separately. I then develop tests for the hypothesis that a multiplicative relationship exists for the risks, i.e., that the combined risk is consistent with the multiplication of the individual risks. Following standard practice in epidemiological studies I consider tests for both case-referent and cohort (standardized mortality rate) type studies.
\end{abstract}

JEL classification numbers:

Key words: synergy, cohort, case-control, epidemiology 


\title{
Statistical Analysis of the Additive and Multiplicative Hypotheses of Multiple Exposure Synergy for Cohort and Case-Control Studies
}

\author{
Jeffrey A. Dubin
}

\section{Introduction}

In epidemiological studies, where there are multiple causes of a particular disease, the issue arises as to whether the multiple causes have a synergistic relationship so that their combined effect is both greater than that of either activity alone, and greater than what one would expect by the sum of their individual risk contributions. Two hypotheses are frequently tested. The first hypothesis states that when the sources of disease act independently, the relative risk of disease, given exposure, is an additive relationship. Thus, the relative risk of dying from cause $A$ adds to the relative risk of dying from cause $B$ to determine the combined relative risk of dying when exposed to both $A$ and $B$. A second hypothesis states that the relationship between disease and the two causal factors is multiplicative. In this case, the combined risk is the product of the individual risks.

Of course synergism is itself a concept that is model dependent. For instance, a lack of synergism in a logit model of risk, as demonstrated by the statistical insignificance of an interaction term, leads to a multiplicative model of relative risk. Consider the following example.

Suppose that the probability of dying from a disease depends on two factors, $A$ and $B$. Let $\delta_{A}$ denote exposure to $A$, and $\delta_{B}$ denote exposure to $B$. Suppose further that the probability of dying is logistic and given by:

$$
P\left[D \mid \delta_{A}, \delta_{B}\right]=1 /\left(1+e^{-\left(X_{0} \beta_{0}+\delta_{A} X_{A} \beta_{A}+\delta_{B} X_{B} \beta_{B}+\delta_{A} \delta_{B} X_{C}\right)}\right)
$$

where $X_{A}, X_{B}, X_{C}$, and $X_{0}$ are vectors of explanatory factors, and $\beta_{j}$ are true but unknown coefficient vectors. The presence of the term $\delta_{A} \delta_{B}$ allows for synergism in this model, and specifies that the probability of disease may be different when causal factors $A$ and $B$ are both present. 
Now, assume that $\beta_{C}$ is zero so that there is no synergistic relationship in the model. The relative odds of dying when exposed to both agents are:

$$
\begin{aligned}
R O_{A B} & =P\left[D \mid \delta_{A}=1, \delta_{B}=1\right] / P\left[\bar{D} \mid \delta_{A}=1, \delta_{B}=1\right] \\
& =\exp \left(X_{0} \beta_{0}+X_{A} \beta_{A}+X_{B} \beta_{B}\right)
\end{aligned}
$$

Similarly, the relative odds of dying when exposed to $A$ alone are:

$$
\begin{aligned}
R O_{A} & =P\left[D \mid \delta_{A}=1, \delta_{B}=0\right] / P\left[\bar{D} \mid \delta_{A}=1 \delta_{B}=0\right] \\
& =\exp \left(X_{0} \beta_{0}+X_{A} \beta_{A}\right)
\end{aligned}
$$

and

$$
\begin{aligned}
R O_{B} & =P\left[D \mid \delta_{A}=0, \delta_{B}=1\right] / P\left[\bar{D} \mid \delta_{A}=0, \delta_{B}=1\right] \\
& =\exp \left(X_{0} \beta_{0}+X_{B} \beta_{B}\right)
\end{aligned}
$$

and the relative odds of dying from background exposure is

$$
\begin{aligned}
R O_{0} & =P\left[D \mid \delta_{A}=0, \delta_{B}=0\right] / P\left[\bar{D} \mid \delta_{A}=0, \delta_{B}=0\right] \\
& =\exp \left(X_{0} \beta_{0}\right)
\end{aligned}
$$

The relative risk is defined as the ratio of the relative odds between the exposure group and the baseline:

$$
\begin{aligned}
R R_{A B} & =\left(\frac{P\left[D \mid \delta_{A}=1, \delta_{B}=1\right]}{P\left[\bar{D} \mid \delta_{A}=1, \delta_{B}=1\right]}\right) /\left(\frac{P\left[D \mid \delta_{A}=0, \delta_{B}=0\right]}{P\left[\bar{D} \mid \delta_{A}=0, \delta_{B}=0\right]}\right) \\
& =\left(\frac{P\left[D \mid \delta_{A}=1, \delta_{B}=1\right]}{P\left[D \mid \delta_{A}=0, \delta_{B}=0\right]}\right) /\left(\frac{P\left[\bar{D} \mid \delta_{A}=1, \delta_{B}=1\right]}{P\left[\bar{D} \mid \delta_{A}=0, \delta_{B}=0\right]}\right)
\end{aligned}
$$

which says that the relative risk from combined exposure is equal to the ratio of the relative odds of dying in the exposed population to the relative odds of dying in the un-exposed population.

$$
\text { Then } \begin{aligned}
R R_{A B} & =\left(R O_{A B} / R O_{0}\right) \\
& =\exp \left(X_{A} \beta_{A}+X_{B} \beta_{B}\right) \text { and } \\
R R_{A} & =\left(R O_{A} / R O_{0}\right)=\exp \left(X_{A} \beta_{A}\right), \quad R R_{B}=\left(R O_{B} / R O_{0}\right)=\exp \left(X_{B} \beta_{B}\right)
\end{aligned}
$$

We see that $R R_{A B}=R R_{A} \cdot R R_{B}$ even though the model exhibits synergism.

This paper considers several methods for determining the relative odds ratio, including the case-control method and the cohort method. The case-control method begins with a group of individuals who have an observed attribute (such as a given disease or death). To the cases are matched a set of control individuals. The matching typically is done at the individual level. For cases and controls, a retrospective determination is made of exposure to one or more contaminants. From the retrospective exposure, prospective odds of becoming a case given exposure are determined. 
Cohort studies, by contrast, derive mortality and morbidity rates with reference to an external reference group. The method is based on the idea of comparing the incidence of disease in an exposed cohort to the number expected in a "normal" reference group.

Finally, relative risks are sometimes determined using the prevalence method. In prevalence studies it is common to analyze populations that all have a common exposure level to some contaminant. In asbestosis studies, it is necessary that all subjects, by definition, have the same exposure to asbestos. In such cases, the issues of additivity and multiplicativity are not germane because one can consider the separate effect of each causal agent. A similar situation occurs in a cohort setting where a companion population is not used as a reference group. These situations are nevertheless illuminating in discerning the relative contribution of a second contaminant as it affects the probability of contracting or dying from a disease. Another example is the analysis of the prevalence of a disease attribute (such as pleural plaques) in an exposed population.

A prevalence model may be fitted with a logistic functional form. The outcome variable is usually the presence or absence of a disease characteristic where the explanatory factors will include control variables and an indicator for the level of contaminant. If the cohort provides some level of variance in the level of exposure of both contaminants, an interactive term can be used to test for synergy, even if this does not provide a test of additivity or multiplicativity.

This paper focuses on testing the statistical hypotheses of additivity and multiplicativity for the relative risk measures. While other papers have considered the confidence intervals for relative risk measures, no systematic study has been made of the additivity and multiplicativity hypotheses as a matter of statistics. Therefore, while practice in epidemiology has been to say that one or more studies appear to support the multiplicative model, these studies have not, in general, been statistical statements; i.e., statements made with attendant levels of confidence.

This paper is divided into six sections. In Section 2, we discuss the case-control method and Wald type tests for the multiplicative and additive hypotheses, derive and discuss Woolf's method for determining the variance of log-odds ratios (Woolf (1955)), and discuss maximum likelihood methods for optimization subject to constraints following the methods of Gardner and Munford (1980). In Section 3, we discuss other approaches for determining confidence intervals, including Bonferroni Intervals and Monte Carlo simulation. In Section 4, we describe various synergy indices and how they relate to tests of hypotheses for additive and multiplicative statistics. In Section 5, we discuss cohort studies and derive hypothesis tests for the additive and multiplicative statistics. In Section 6, we discuss prevalence studies and their relationship to cohort studies. In Section 7, we present our conclusions. 


\section{Case-Control Studies}

We begin with a table of case-control outcomes at differing exposure levels:

\begin{tabular}{|l|c|c|c|c|c|}
\hline \multicolumn{7}{|c|}{ Exposure } \\
\hline & None & $A$ & $B$ & $A \& B$ & TOTAL \\
\hline cases & $h_{1}$ & $h_{2}$ & $h_{3}$ & $h_{4}$ & $h$ \\
controls & $k_{1}$ & $k_{2}$ & $k_{3}$ & $k_{4}$ & $k$ \\
\hline
\end{tabular}

We next express the row counts as fractions:

\begin{tabular}{|l|c|c|c|c|c|}
\hline \multicolumn{7}{|c|}{ Exposure } \\
\hline & None & $A$ & $B$ & $A \& B$ & TOTAL \\
\hline cases & $\pi_{1}$ & $\pi_{2}$ & $\pi_{3}$ & $\pi_{4}$ & 1 \\
controls & $\theta_{1}$ & $\theta_{2}$ & $\theta_{3}$ & $\theta_{4}$ & 1 \\
\hline
\end{tabular}

where $\hat{\pi}_{j}=h_{j} / h$ and $\hat{\theta}_{j}=k_{j} / k$ are consistent estimates of the true cell probabilities.

First, we demonstrate that the retrospective odds-ratio from a case control method provides an approximate estimate of the relative risk of being a "case," given exposure. To prove this, we examine the odds ratio $\pi_{4} \cdot \theta_{1} / \pi_{1} \cdot \theta_{4}$, although the result clearly generalizes to other cases. We show that, given exposure level $A \& B$, the odds-ratio approximates the relative risk of being a case.

We denote cases as $D$ (death from lung cancer for instance) and $\bar{D}$ a control (death from other causes for instance). The combined exposure $A \& B$ is referred to as $E$ (exposure). A case with no exposure is denoted $\bar{E}$ (no exposure).

The odds-ratio $\pi_{4} \cdot \theta_{1} / \pi_{1} \cdot \theta_{4}$ is equal to

$$
\frac{P[E \mid D] \cdot P[\bar{E} \mid \bar{D}]}{P[\bar{E} \mid D] \cdot P[E \mid \bar{D}]}
$$

since $\pi_{4}=P[E \mid D], \pi_{1}=P[\bar{E} \mid D], \theta_{4}=P[E \mid \bar{D}]$, and $\theta_{1}=P[\bar{E} \mid \bar{D}]$

As the notation implies, the probabilities $\pi$ and $\theta$ are conditional probabilities indicating the respective likelihood of having been exposed, given an individual's case-control status. Of interest is the prospective probability of being a case (i.e., dying) given exposure status. ${ }^{1}$

\footnotetext{
${ }^{1}$ Some research studies have used logit analysis to model the conditional probabilities shown above. This allows the introduction of covariates to provide additional controls in the analysis. For example a logit model may be used to specify the conditional probabilities: $P[A \mid D], P[\bar{A} \mid D], P[A \mid \bar{D}]$, and $P[\bar{A} \mid \bar{D}$. A specification of such a model was illustrated in the introduction. The presence of additional covariates complicates the analysis presented below as the variances and covariances become dependent on the assumed probability model and on the precision of the parameter estimation.
} 
Under a simplifying assumption, the odds-ratio approximates the prospective odds:

$$
\begin{aligned}
\frac{P[E \mid D] \cdot P[\bar{E} \mid \bar{D}]}{P[\bar{E} \mid D] \cdot P[E \mid \bar{D}]} & =\frac{P[E, D] / P[D]}{P[E, \bar{D}] / P[\bar{D}]} \cdot \frac{P[\bar{E}, \bar{D}] / P[\bar{D}]}{P[\bar{E}, D] / P[D]} \\
& =\frac{P[D \mid E] \cdot P[E]}{P[D \mid \bar{E}] \cdot P[\bar{E}]} \cdot \frac{P[\bar{D} \mid \bar{E}] \cdot P[\bar{E}]}{P[\bar{D} \mid E] \cdot P[E]} \\
& =\frac{P[D \mid E]}{P[D \mid \bar{E}]} \cdot \frac{P[\bar{D} \mid \bar{E}]}{P[\bar{D} \mid E]} \\
& \doteq \frac{P[D \mid E]}{P[D \mid \bar{E}]}
\end{aligned}
$$

where the approximation results from the observation that $P[\bar{D} \mid \bar{E}] / P[\bar{D} \mid E]$ is close to one. Case control studies are useful as they provide estimates of the odds $P[E] / P[\bar{E}]$; i.e., the relative odds of exposure. The relative odds of being a case $P[D] / P[\bar{D}]$ are irrelevant as they are set by the researcher in the design. They do, however, have an influence on the confidence of the results.

\subsection{Hypothesis Tests for Case-Control Studies-Multiplicative Case}

The relative risk (prospective) of dying given exposure to contaminant $A$ is $\pi_{2} \theta_{1} / \pi_{1} \theta_{2}$. The relative risk of dying given exposure to contaminant $B$ is $\pi_{3} \theta_{1} / \pi_{1} \theta_{3}$. The relative risk of dying if exposed to both contaminants is $\pi_{4} \theta_{1} / \pi_{1} \theta_{4}$. The multiplicative hypothesis states that $R R_{A \& B}=R R_{A} \cdot R R_{B}$ so that:

$$
\pi_{4} \theta_{1} / \pi_{1} \theta_{4}=\left(\pi_{2} \theta_{1} / \pi_{1} \theta_{2}\right) \cdot\left(\pi_{3} \theta_{1} / \pi_{1} \theta_{3}\right)
$$

Taking logarithms, this becomes:

$\log \pi_{4}+\log \theta_{1}-\log \pi_{1}-\log \theta_{4}-\log \pi_{2}-\log \theta_{1}+\log \pi_{1}+\log \theta_{2}-\log \pi_{3}-\log \theta_{1}+\log \pi_{1}+\log \theta_{3}=0$

This may be rewritten as

$$
\log \left(\pi_{4} \theta_{2} \theta_{3} \pi_{1}\right)-\log \left(\theta_{4} \pi_{2} \pi_{3} \theta_{1}\right)=0
$$

or

$$
M=\left(\log \pi_{1}-\log \pi_{2}-\log \pi_{3}+\log \pi_{4}\right)-\left(\log \theta_{1}-\log \theta_{2}-\log \theta_{3}+\log \theta_{4}\right)=0
$$

A consistent estimate of this statistic is obtained by replacing $\pi_{j}$ and $\theta_{j}$ with $\hat{\pi}_{j}$ and $\hat{\theta}_{j}$.

Deriving the variance of the resulting statistic is complicated by the fact that $h_{1}$, $h_{2}, h_{3}$, and $h_{4}$ form a multinomial probability distribution. Similarly $k_{1}, k_{2}, k_{3}$, and $k_{4}$ form a multinomial probability distribution, but one which is independent of the joint distribution of the $h_{j} k_{j}$ assumption.

To derive the joint distribution of the $\log \pi_{j}$ and $\log \theta_{j}$, we begin with results for the joint distribution of the $h_{j}$. Similar results hold for the outcome of the $k_{j}$. For notational simplicity we present the results using a common symbol $n_{j}$ where $n_{1}+n_{2}+n_{3}+n_{4}=n$. 
Lemma 1 Let $\delta_{j t}=1$ if outcome $j$ is realized in observation $t$. The probability that $\delta_{j t}=1$ is denoted $\pi_{j}$. Let $n_{j}$ denote the total number of outcome $j$ 's that are observed in the sample of $n$ independent draws, with

$$
\begin{gathered}
\pi_{1}+\pi_{2}+\pi_{3}+\pi_{4}=1, \quad n_{1}+n_{2}+n_{3}+n_{4}=n, \\
n_{j}=\sum_{t=1}^{n} \delta_{j t}, \quad n=\sum_{t=1}^{n}\left(\delta_{1 t}+\delta_{2 t}+\delta_{3 t}+\delta_{4 t}\right)=\sum_{t=1}^{n} 1 .
\end{gathered}
$$

Then $E\left(n_{j}\right)=n \pi_{j}, V\left(n_{j}\right)=n \pi_{j}\left(1-\pi_{j}\right)$, and $\operatorname{cov}\left(n_{j}, n_{k}\right)=-n \pi_{j} \pi_{k}$ for $j \neq k$.

Proof: $n_{j}=\sum_{t=1}^{n} \delta_{j t}$ implies $E\left(n_{j}\right)=\sum_{t=1}^{n} E\left(\delta_{j t}\right)=n \pi_{j}$ since $E\left(\delta_{j}\right)=1 \cdot \pi_{j}+0 \cdot\left(1-\pi_{j}\right)$. Next $V\left(n_{j}\right)=\sum_{t=1}^{n} V\left(\delta_{j t}\right)$. But $V\left(\delta_{j t}\right)=E\left(\delta_{j t}\right)-E\left(\delta_{j t}\right)^{2}=\pi_{j}-\pi_{j}^{2}=\pi_{j}\left(1-\pi_{j}\right)$. Hence $V\left(n_{j}\right)=n \pi_{j}\left(1-\pi_{j}\right)$. Finally $\operatorname{cov}\left(n_{j}, n_{k}\right)=E\left[\left(n_{j}-n \pi_{j}\right)\left(n_{k}-n \pi_{k}\right)\right]=E\left(n_{j} n_{k}\right)-n \pi_{j} n \pi_{k}-$ $n \pi_{k} n \pi_{j}+n^{2} \pi_{j} \pi_{k}=E\left(n_{j} n_{k}\right)-n^{2} \pi_{j} \pi_{k}$. Now

$$
E\left(n_{j} n_{k}\right)=E\left[\left(\sum_{t} \delta_{j t}\right)\left(\sum_{t} \delta_{k t}\right)\right]=E\left[\sum_{t} \delta_{j t} \delta_{k t}+\sum_{t \neq s} \delta_{j t} \delta_{k s}\right]
$$

But $\delta_{j t} \delta_{k t}=0$ if $j \neq k$ in observation $t$ (only one unique outcome is realized in each trial) so that the first sum is exactly zero. The second sum consists of $\left(n^{2}-n\right)$ terms, which are the products of independent random variables (since $\delta_{j t}$ and $\delta_{k s}$ are independent when $t \neq s)$. The expectation of each term in the second sum is $E\left(\delta_{j t} \delta_{k s}\right)=\pi_{j} \pi_{k}$.

Hence $E\left(n_{j} n_{k}\right)=\left(n^{2}-n\right) \pi_{j} \pi_{k}$. Combining these results we obtain

$$
\begin{aligned}
\operatorname{cov}\left(n_{j}, n_{k}\right) & =\left(n^{2}-n\right) \pi_{j} \pi_{k}-n^{2} \pi_{j} \pi_{k} \\
& =-n \pi_{j} \pi_{k}
\end{aligned}
$$

Combining these results into the variance covariance matrix for $n_{j}$ we obtain:

$$
E\left(\begin{array}{l}
n_{1} \\
n_{2} \\
n_{3} \\
n_{4}
\end{array}\right)=n\left(\begin{array}{l}
\pi_{1} \\
\pi_{2} \\
\pi_{3} \\
\pi_{4}
\end{array}\right)
$$

and

$$
V\left(\begin{array}{c}
n_{1} \\
n_{2} \\
n_{3} \\
n_{4}
\end{array}\right)=n\left(\begin{array}{cccc}
\pi_{1}\left(1-\pi_{1}\right) & -\pi_{1} \pi 2 & -\pi_{1} \pi_{3} & -\pi_{1} \pi_{4} \\
-\pi_{2} \pi_{1} & \pi_{2}\left(1-\pi_{2}\right) & -\pi_{2} \pi_{3} & -\pi_{2} \pi_{4} \\
-\pi_{3} \pi_{1} & -\pi_{3} \pi_{2} & \pi_{3}\left(1-\pi_{3}\right) & -\pi_{3} \pi_{4} \\
-\pi_{4} \pi_{1} & -\pi_{4} \pi_{2} & -\pi_{4} \pi_{3} & \pi_{4}\left(1-\pi_{4}\right)
\end{array}\right)=n\left(I-\phi \phi^{\prime}\right)
$$

where $\phi=\left(\begin{array}{llll}\sqrt{\pi_{1}} & \sqrt{\pi_{2}} & \sqrt{\pi_{3}} & \sqrt{\pi_{4}}\end{array}\right)^{\prime}$. 
To derive the variance-covariance matrix for $\log \hat{\pi}_{j}=\log \left(n_{j} / n\right)$, we use a Taylor's series expansion to first-order for the logarithm. Then

$$
\log \hat{\pi}_{j} \doteq \log \pi_{j}+\frac{1}{\pi_{j}}\left(\hat{\pi}_{j}-\pi_{j}\right)
$$

where we have evaluated the Taylor's expansion around the true but unknown $\pi_{j}$. Then

$$
\log \left(\begin{array}{c}
\hat{\pi}_{1} \\
\hat{\pi}_{2} \\
\hat{\pi}_{3} \\
\hat{\pi}_{4}
\end{array}\right)=\left(\begin{array}{l}
\log \pi_{1} \\
\log \pi_{2} \\
\log \pi_{3} \\
\log \pi_{4}
\end{array}\right)+\left(\begin{array}{cccc}
1 / \pi_{1} & & 0 & \\
& 1 / \pi_{2} & & \\
0 & & 1 / \pi_{3} & \\
& & & 1 / \pi_{4}
\end{array}\right)\left(\begin{array}{c}
\left(\hat{\pi}_{1}-\pi_{1}\right) \\
\left(\hat{\pi}_{2}-\pi_{2}\right) \\
\left(\hat{\pi}_{3}-\pi_{3}\right) \\
\left(\hat{\pi}_{4}-\pi_{4}\right)
\end{array}\right)
$$

Hence

$$
\begin{aligned}
& V\left(\log \hat{\pi}_{j}\right)=\left(\begin{array}{cccc}
1 / \pi_{1} & & 0 & \\
& 1 / \pi_{2} & & \\
0 & & 1 / \pi_{3} & \\
& & & 1 / \pi_{4}
\end{array}\right) \operatorname{Var}\left(\hat{\pi}_{j}-\pi_{j}\right)\left(\begin{array}{cccc}
1 / \pi_{1} & & & \\
& 1 / \pi_{2} & & \\
& & 1 / \pi_{3} & \\
& & & 1 / \pi_{4}
\end{array}\right)^{\prime} \\
& =\frac{1}{n}\left(\begin{array}{ccccc}
1 / \pi_{1} & & 0 & \\
& 1 / \pi_{2} & & \\
0 & & 1 / \pi_{3} & \\
& & & 1 / \pi_{4}
\end{array}\right)\left(\begin{array}{cccc}
\pi_{1}\left(1-\pi_{1}\right) & -\pi_{1} \pi 2 & -\pi_{1} \pi_{3} & -\pi_{1} \pi_{4} \\
-\pi_{2} \pi_{1} & \pi_{2}\left(1-\pi_{2}\right) & -\pi_{2} \pi_{3} & -\pi_{2} \pi_{4} \\
-\pi_{3} \pi_{1} & -\pi_{3} \pi_{2} & \pi_{3}\left(1-\pi_{3}\right) & -\pi_{3} \pi_{4} \\
-\pi_{4} \pi_{1} & -\pi_{4} \pi_{2} & -\pi_{4} \pi_{3} & \pi_{4}\left(1-\pi_{4}\right)
\end{array}\right) . \\
& \left(\begin{array}{cccc}
1 / \pi_{1} & & 0 & \\
& 1 / \pi_{2} & & \\
0 & & 1 / \pi_{3} & \\
& & & 1 / \pi_{4}
\end{array}\right)^{\prime} \\
& \text { since } \operatorname{Var}\left(\hat{\pi}_{j}\right)=\frac{1}{n^{2}} \operatorname{Var}\left(n_{j}\right)=\frac{1}{n}\left(\begin{array}{cccc}
\pi_{1}\left(1-\pi_{1}\right) & -\pi_{1} \pi 2 & -\pi_{1} \pi_{3} & -\pi_{1} \pi_{4} \\
-\pi_{2} \pi_{1} & \pi_{2}\left(1-\pi_{2}\right) & -\pi_{2} \pi_{3} & -\pi_{2} \pi_{4} \\
-\pi_{3} \pi_{1} & -\pi_{3} \pi_{2} & \pi_{3}\left(1-\pi_{3}\right) & -\pi_{3} \pi_{4} \\
-\pi_{4} \pi_{1} & -\pi_{4} \pi_{2} & -\pi_{4} \pi_{3} & \pi_{4}\left(1-\pi_{4}\right)
\end{array}\right) \text {. }
\end{aligned}
$$

Next $n V\left(\log \hat{\pi}_{j}\right)$

$$
\begin{aligned}
& =\left(\begin{array}{ccccc}
1 / \pi_{1} & & 0 & \\
& 1 / \pi_{2} & & \\
0 & & 1 / \pi_{3} & \\
& & & 1 / \pi_{4}
\end{array}\right)\left(\begin{array}{cccc}
\left(1-\pi_{1}\right) & -\pi_{1} & -\pi_{1} & -\pi_{1} \\
-\pi_{2} & \left(1-\pi_{2}\right) & -\pi_{2} & -\pi_{2} \\
-\pi_{3} & -\pi_{3} & \left(1-\pi_{3}\right) & -\pi_{3} \\
-\pi_{4} & -\pi_{4} & -\pi_{4} & \left(1-\pi_{4}\right)
\end{array}\right) \\
& =\left(\begin{array}{ccccc}
\left(1-\pi_{1}\right) / \pi_{1} & -1 & -1 & -1 \\
-1 & \left(1-\pi_{2}\right) / \pi_{2} & -1 & -1 \\
-1 & -1 & \left(1-\pi_{3}\right) / \pi_{3} & -1 \\
-1 & -1 & -1 & \left(1-\pi_{4}\right) / \pi_{4}
\end{array}\right)
\end{aligned}
$$


Theorem 2 For the multiplicative hypothesis,

$$
\operatorname{Var}(M)=\left(\frac{1}{h_{1}}+\frac{1}{h_{2}}+\frac{1}{h_{3}}+\frac{1}{h_{4}}\right)+\left(\frac{1}{k_{1}}+\frac{1}{k_{2}}+\frac{1}{k_{3}}+\frac{1}{k_{4}}\right)
$$

Proof: The multiplicative hypothesis may be written as

$$
M=\left(\begin{array}{llll}
1 & -1 & -1 & 1
\end{array}\right)\left(\begin{array}{l}
\log \hat{\pi}_{1} \\
\log \hat{\pi}_{2} \\
\log \hat{\pi}_{3} \\
\log \hat{\pi}_{4}
\end{array}\right)-\left(\begin{array}{llll}
1 & -1 & -1 & 1
\end{array}\right)\left(\begin{array}{l}
\log \hat{\theta}_{1} \\
\log \hat{\theta}_{2} \\
\log \hat{\theta}_{3} \\
\log \hat{\theta}_{4}
\end{array}\right)
$$

Hence

$$
\begin{aligned}
& \operatorname{Var}(M)=\frac{1}{h}\left(\begin{array}{llll}
1 & -1 & -1 & 1
\end{array}\right) \\
& \left(\begin{array}{cccc}
\left(1-\pi_{1}\right) / \pi_{1} & -1 & -1 & -1 \\
-1 & \left(1-\pi_{2}\right) / \pi_{2} & -1 & -1 \\
-1 & -1 & \left(1-\pi_{3}\right) / \pi_{3} & -1 \\
-1 & -1 & -1 & \left(1-\pi_{4}\right) / \pi_{4}
\end{array}\right)\left(\begin{array}{r}
1 \\
-1 \\
-1 \\
1
\end{array}\right) \\
& +\frac{1}{k}\left(\begin{array}{llll}
1 & -1 & -1 & 1
\end{array}\right) \\
& \left(\begin{array}{cccc}
\left(1-\theta_{1}\right) / \theta_{1} & -1 & -1 & -1 \\
-1 & \left(1-\theta_{2}\right) / \theta_{2} & -1 & -1 \\
-1 & -1 & \left(1-\theta_{3}\right) / \theta_{3} & -1 \\
-1 & -1 & -1 & \left(1-\theta_{4}\right) / \theta_{4}
\end{array}\right)\left(\begin{array}{r}
1 \\
-1 \\
-1 \\
1
\end{array}\right) \\
& \frac{1}{h}\left[\quad(1) \quad\left[\left(1-\pi_{1}\right) / \pi_{1}+1+1-1\right]+\right. \\
& =\quad \begin{array}{lll}
(-1) & {\left[-1-\left(1-\pi_{2}\right) / \pi_{2}+1-1\right]+} \\
(-1) & {\left[-1+1-\left(1-\pi_{3}\right) / \pi_{3}-1\right]+}
\end{array} \\
& \text { (1) } \left.\left[-1+1+1+\left(1-\pi_{4}\right) / \pi_{4}\right] \quad\right]+ \text { similar terms in } \theta \\
& =\frac{1}{h}\left[\frac{\left(1-\pi_{1}\right)}{\pi_{1}}+1+\frac{\left(1-\pi_{2}\right)}{\pi_{2}}+1+\frac{\left(1-\pi_{3}\right)}{\pi_{3}}+1+\frac{\left(1-\pi_{4}\right)}{\pi_{4}}+1\right]+ \\
& \text { similar terms in } \theta \\
& =\frac{1}{h}\left[\frac{1}{\pi_{1}}+\frac{1}{\pi_{2}}+\frac{1}{\pi_{3}}+\frac{1}{\pi_{4}}\right]+\text { similar terms in } \theta
\end{aligned}
$$

Hence

$$
\begin{aligned}
\operatorname{Var}(M) & =\left[\left(\frac{1}{h \pi_{1}}+\frac{1}{h \pi_{2}}+\frac{1}{h \pi_{3}}+\frac{1}{h \pi_{4}}\right)+\left(\frac{1}{k \theta_{1}}+\frac{1}{k \theta_{2}}+\frac{1}{k \theta_{3}}+\frac{1}{k \theta_{4}}\right)\right] \\
& =\left(\frac{1}{h_{1}}+\frac{1}{h_{2}}+\frac{1}{h_{3}}+\frac{1}{h_{4}}\right)+\left(\frac{1}{k_{1}}+\frac{1}{k_{2}}+\frac{1}{k_{3}}+\frac{1}{k_{4}}\right) .
\end{aligned}
$$




\subsection{Woolf's Method}

A similar result for the variance of a log odds-ratio itself is derived as follows. Consider $\log \left(\pi_{4} \theta_{1} / \pi_{1} \theta_{4}\right)$, the log-odds ratio for the relative risk at the combined exposure level in a case control study. We have

$$
\log R R_{A \& B}=\log \left(\pi_{4} \theta_{1} / \pi_{1} \theta_{4}\right)=\log \left(\pi_{4} / \pi_{1}\right)-\log \left(\theta_{4} / \theta_{1}\right)
$$

Next, without loss of generality, assume that $\pi_{1}$ and $\pi_{4}$ have been normalized so that $\pi_{1}+\pi_{4}=1$ (This may be accomplished by setting $\pi_{1}^{\prime}=\pi_{1} /\left(\pi_{1}+\pi_{4}\right)$ and $\pi_{4}^{\prime}=\pi_{4} /\left(\pi_{1}+\pi_{4}\right)$. Now $\pi_{1}^{\prime}+\pi_{4}^{\prime}=1$ and the log odds-ratio remains unchanged since

$$
\log R R_{A \& B}=\log \left(\pi_{4}^{\prime} \theta_{1}^{\prime} / \pi_{1}^{\prime} \theta_{4}^{\prime}\right)=\log \left(\pi_{4} \theta_{1} / \pi_{1} \theta_{4}\right) .
$$

The expression for $\log \left(\pi_{4} / \pi_{1}\right)$ is in the form $\log \left(\frac{\rho}{1-\rho}\right)$ where $\rho=\pi_{4}$ and $(1-\rho)=\pi_{1}$. A Taylor's series expansion of $\log \left(\frac{\rho}{1-\rho}\right)$ demonstrates that:

$$
\begin{aligned}
\log \frac{\rho}{1-\rho} & =\log \frac{\rho_{0}}{1-\rho_{0}}+\left.\frac{1-\rho}{\rho}\left[\frac{(1-\rho)+\rho}{(1-\rho)^{2}}\right]\right|_{\rho_{0}} \cdot\left(\rho-\rho_{0}\right) \\
& =\log \frac{\rho_{0}}{1-\rho_{0}}+\frac{\rho-\rho_{0}}{\rho_{0}\left(1-\rho_{0}\right)} .
\end{aligned}
$$

Next

$$
\begin{aligned}
\operatorname{Var}\left(\log \frac{\hat{\rho}}{(1-\hat{\rho})}\right) & =\left(\frac{1}{\hat{\rho}(1-\hat{\rho})}\right)^{2} \frac{\hat{\rho}(1-\hat{\rho})}{N} \\
& =\frac{1}{N \hat{\rho}(1-\hat{\rho})}
\end{aligned}
$$

where $\hat{\rho}=\frac{1}{N} \sum_{t=1}^{N} \delta_{t}$ is the unbiased estimator of $\rho, E(\hat{\rho})=\rho$ and $\operatorname{Var}(\hat{\rho})=\hat{\rho}(1-$ $\hat{\rho}) / N$ and $N$ is the number of independent trials resulting in $\sum_{t=1}^{N} \delta_{t}$ exposure cases (as compared to non-exposure cases). Similar expressions follow for the theta distribution. Now

$$
\begin{aligned}
\operatorname{Var}\left(\log \left(\hat{\pi}_{4} / \hat{\pi}_{1}\right)\right) & =\frac{1}{h \hat{\pi}_{4} \hat{\pi}_{1}}=\frac{h}{\left(h \hat{\pi}_{4}\right)\left(h \hat{\pi}_{1}\right)} \\
& =\frac{h_{1}+h_{4}}{h_{1} h_{4}} \\
& =\frac{1}{h_{4}}+\frac{1}{h_{1}} .
\end{aligned}
$$

Then

$$
\operatorname{Var}\left(\log R R_{A \& B}\right)=\frac{1}{h_{1}}+\frac{1}{h_{4}}+\frac{1}{k_{1}}+\frac{1}{k_{4}}
$$


Note that the repeated application of this result (assuming independence) to the multiplicative hypothesis would not produce the correct result in a case control setting because $R R_{A \& B}, R R_{A}$ and $R R_{B}$ are mutually correlated.

This result is also known as Woolf's method, and is sometimes written

$$
\operatorname{Var}\left(\log \frac{A D}{B C}\right)=\frac{1}{A}+\frac{1}{B}+\frac{1}{C}+\frac{1}{D}
$$

where $R R=A D / B C$ and $A$ denotes the number of cases with exposure, $B$ denotes cases without exposure, $C$ denotes controls with exposure, and $D$ denotes controls without exposure.

It is also possible to derive the covariances of the relative risk measures. Consider $R R_{A}=\pi_{2} \theta_{1} / \pi_{1} \theta_{2}$ and $R R_{A \& B}=\pi_{4} \theta_{1} / \pi_{1} \theta_{4}$. Then

$$
\log R R_{A}=\left(\log \pi_{2}-\log \pi_{1}\right)-\left(\log \theta_{2}-\log \theta_{1}\right)
$$

and

$$
\log R R_{A \& B}=\left(\log \pi_{4}-\log \pi_{1}\right)-\left(\log \theta_{4}-\log \theta_{1}\right) .
$$

Clearly, these are correlated because of the common components. Consider the $\pi$ components first (analogous results apply to the $\theta$ components). Recall that

$$
\operatorname{Var}(\log \hat{\pi})=\frac{1}{h}\left(\begin{array}{cccc}
\left(1-\pi_{1}\right) / \pi_{1} & -1 & -1 & -1 \\
-1 & \left(1-\pi_{2}\right) / \pi_{2} & -1 & -1 \\
-1 & -1 & \left(1-\pi_{3}\right) / \pi_{3} & -1 \\
-1 & -1 & -1 & \left(1-\pi_{4}\right) / \pi_{4}
\end{array}\right)
$$

But $\quad \log \pi_{2}-\log \pi_{1}=\left(\begin{array}{llll}-1 & 1 & 0 & 0\end{array}\right)\left[\begin{array}{l}\log \pi_{1} \\ \log \pi_{2} \\ \log \pi_{3} \\ \log \pi_{4}\end{array}\right] \quad$ so that

$\operatorname{Var}\left(\log \pi_{2}-\log \pi_{1}\right)=$

$$
\begin{aligned}
& \left(\frac{1}{h}\right)\left(\begin{array}{llll}
-1 & 1 & 0 & 0
\end{array}\right) \\
& \left(\begin{array}{cccc}
\left(1-\pi_{1}\right) / \pi_{1} & -1 & -1 & -1 \\
-1 & \left(1-\pi_{2}\right) / \pi_{2} & -1 & -1 \\
-1 & -1 & \left(1-\pi_{3}\right) / \pi_{3} & -1 \\
-1 & -1 & -1 & \left(1-\pi_{4}\right) / \pi_{4}
\end{array}\right)\left(\begin{array}{r}
-1 \\
1 \\
0 \\
0
\end{array}\right) \\
= & \left(\frac{1}{h}\right)\left(\begin{array}{llll}
-1 & 1 & 0 & 0
\end{array}\right)\left[\begin{array}{c}
-\left(1-\pi_{1}\right) / \pi_{1}-1 \\
1+\left(1-\pi_{2}\right) / \pi_{2} \\
0 \\
0
\end{array}\right] \\
= & \frac{1}{h}\left(\frac{\left(1-\pi_{1}\right)}{\pi_{1}}+1+\frac{\left(1-\pi_{2}\right)}{\pi_{2}}+1\right) \\
= & \left(\frac{1}{h}\right)\left(\frac{1}{\pi_{1}}\right)+\left(\frac{1}{h}\right)\left(\frac{1}{\pi_{2}}\right)=\frac{1}{h_{1}}+\frac{1}{h_{2}}
\end{aligned}
$$


Combining this with the analogous result for $\log \theta_{2}-\log \theta_{1}$, we obtain:

$$
\operatorname{Var}\left(\log R R_{A}\right)=\frac{1}{h_{1}}+\frac{1}{h_{2}}+\frac{1}{k_{1}}+\frac{1}{k_{2}}
$$

This is exactly the Woolf result shown above. Similarly:

$$
\operatorname{Var}\left(\log R R_{A \& B}\right)=\frac{1}{h_{1}}+\frac{1}{h_{4}}+\frac{1}{k_{1}}+\frac{1}{k_{4}}
$$

and

$$
\operatorname{Var}\left(\log R R_{B}\right)=\frac{1}{h_{1}}+\frac{1}{h_{3}}+\frac{1}{k_{1}}+\frac{1}{k_{3}}
$$

Next consider the covariance between $\log R R_{A}$ and $\log R R_{A \& B}$. Again, we consider the $\pi$ terms first. Using the fact that $\operatorname{cov}\left(t^{\prime} x, s^{\prime} x\right)=t^{\prime} \operatorname{Var}(x) s$ for conformable column vectors, we have, (for the $\pi$ terms only)

$$
\begin{aligned}
& \operatorname{cov}\left[\log R R_{A}, \log R R_{B}\right]=\left(\frac{1}{h}\right)\left(\begin{array}{llll}
-1 & 1 & 0 & 0
\end{array}\right) . \\
& \left(\begin{array}{cccc}
\left(1-\pi_{1}\right) / \pi_{1} & -1 & -1 & -1 \\
-1 & \left(1-\pi_{2}\right) / \pi_{2} & -1 & -1 \\
-1 & -1 & \left(1-\pi_{3}\right) / \pi_{3} & -1 \\
-1 & -1 & -1 & \left(1-\pi_{4}\right) / \pi_{4}
\end{array}\right)\left(\begin{array}{r}
-1 \\
0 \\
0 \\
1
\end{array}\right) \\
& =\left(\frac{1}{h}\right)\left(\begin{array}{llll}
-1 & 1 & 0 & 0
\end{array}\right)\left[\begin{array}{r}
-\left(1-\pi_{1}\right) / \pi_{1}-1 \\
1-1 \\
1-1 \\
1+\left(1-\pi_{4}\right) / \pi_{4}
\end{array}\right] \\
& =\left(\frac{1}{h}\right)\left(\frac{1}{\pi_{1}}\right)=\frac{1}{h_{1}}
\end{aligned}
$$

A similar covariance term can be derived for the $\theta$ terms.

Thus $\operatorname{cov}\left[\log R R_{A}, \log R R_{A \& B}\right]=\frac{1}{h_{1}}+\frac{1}{k_{1}}$.

Combining analogous results for all log-odds ratios we obtain:

$$
\operatorname{Var}\left[\begin{array}{l}
\log R R_{A} \\
\log R R_{B} \\
\log R R_{A \& B}
\end{array}\right]=\left[\begin{array}{lll}
\frac{1}{h_{1}}+\frac{1}{h_{2}}+\frac{1}{k_{1}}+\frac{1}{k_{2}} & \frac{1}{h_{1}}+\frac{1}{k_{1}} & \frac{1}{h_{1}}+\frac{1}{k_{1}} \\
\frac{1}{h_{1}}+\frac{1}{k_{1}} & \frac{1}{h_{1}}+\frac{1}{h_{3}}+\frac{1}{k_{1}}+\frac{1}{k_{3}} & \frac{1}{h_{1}}+\frac{1}{k_{1}} \\
\frac{1}{h_{1}}+\frac{1}{k_{1}} & \frac{1}{h_{1}}+\frac{1}{k_{1}} & \frac{1}{h_{1}}+\frac{1}{h_{4}}+\frac{1}{k_{1}}+\frac{1}{k_{4}}
\end{array}\right]
$$

We now apply these results to derive the variance of the multiplicative statistic, $M$. We have

$$
\begin{aligned}
M & =\log R R_{A \& B}-\log R R_{A}-\log R R_{B} \\
& =\left(\begin{array}{lll}
-1 & -1 & 1
\end{array}\right)\left[\begin{array}{l}
\log R R_{A} \\
\log R R_{B} \\
\log R R_{A \& B}
\end{array}\right] .
\end{aligned}
$$


Hence, $\operatorname{Var}(M)=$

$$
\begin{aligned}
& \left(\begin{array}{lll}
-1 & -1 & 1
\end{array}\right)\left[\begin{array}{lll}
\frac{1}{h_{1}}+\frac{1}{h_{2}}+\frac{1}{k_{1}}+\frac{1}{k_{2}} & \frac{1}{h_{1}}+\frac{1}{k_{1}} & \frac{1}{h_{1}}+\frac{1}{k_{1}} \\
\frac{1}{h_{1}}+\frac{1}{k_{1}} & \frac{1}{h_{1}}+\frac{1}{h_{3}}+\frac{1}{k_{1}}+\frac{1}{k_{3}} & \frac{1}{h_{1}}+\frac{1}{k_{1}} \\
\frac{1}{h_{1}}+\frac{1}{k_{1}} & \frac{1}{h_{1}}+\frac{1}{k_{1}} & \frac{1}{h_{1}}+\frac{1}{h_{4}}+\frac{1}{k_{1}}+\frac{1}{k_{4}}
\end{array}\right]\left(\begin{array}{r}
-1 \\
-1 \\
1
\end{array}\right) \\
& =\left(\begin{array}{lll}
-1 & -1 & 1
\end{array}\right)\left[\begin{array}{l}
-\left(\frac{1}{h_{1}}+\frac{1}{h_{2}}+\frac{1}{k_{1}}+\frac{1}{k_{2}}\right) \\
-\left(\frac{1}{h_{1}}+\frac{1}{h_{3}}+\frac{1}{k_{1}}+\frac{1}{k_{3}}\right) \\
\left(\frac{1}{h_{1}}+\frac{1}{h_{4}}+\frac{1}{k_{1}}+\frac{1}{k_{4}}\right)-\left(\frac{1}{h_{1}}+\frac{1}{k_{1}}\right)-\left(\frac{1}{h_{1}}+\frac{1}{k_{1}}\right)
\end{array}\right] \\
& =\left(\frac{1}{h_{1}}+\frac{1}{h_{2}}+\frac{1}{h_{3}}+\frac{1}{h_{4}}\right)+\left(\frac{1}{k_{1}}+\frac{1}{k_{2}}+\frac{1}{k_{3}}+\frac{1}{k_{4}}\right)
\end{aligned}
$$

Hence, this formula for $\operatorname{Var}(\log M)$ agrees with our previous derivation.

To test the multiplicative hypothesis, we note that $\log M$ should be zero if the multiplicative hypothesis is true. Therefore we can perform a Wald test using the ratio of $\log (M)$ to its standard error $\sqrt{\operatorname{Var}(\log M)}$. This will have an asymptotic normal distribution. (Rao, Linear Statistical Inference and its Applications).

\subsection{Hypothesis Tests for Case-Control Studies - Additive Case}

We next consider the additive hypothesis, which may be stated:

$$
A=R R_{A \& B}-\left(R R_{A}+R R_{B}-1\right)=0
$$

i.e., that the relative risk of dying from contaminants $A \& B$ is equal to the sum of the relative risks from $A$ and $B$ separately less one. To derive a variance for the statistic $A$, we note that

$$
\begin{aligned}
\operatorname{Var}(A)= & \operatorname{Var}\left(R R_{A \& B}\right)+\operatorname{Var}\left(R R_{A}\right)+\operatorname{Var}\left(R R_{B}\right) \\
& -2 \operatorname{cov}\left(R R_{A \& B}, R R_{A}+R R_{B}\right) \\
= & \operatorname{Var}\left(R R_{A \& B}\right)+\operatorname{Var}\left(R R_{A}\right)+\operatorname{Var}\left(R R_{B}\right) \\
& +2 \operatorname{cov}\left(R R_{A}, R R_{B}\right)-2 \operatorname{cov}\left(R R_{A \& B}, R R_{A}\right) \\
& -2 \operatorname{cov}\left(R R_{A \& B}, R R_{B}\right)
\end{aligned}
$$

In the derivations presented thus far, we have found expressions for the variances and covariances of $\log$ relative risks. Clearly, the additive hypothesis requires variances and covariances of the relative risks themselves. One approach is to develop confidence intervals for the log relative risks, and translate them into confidence intervals for the relative risks by exponentiating the terms in the confidence interval inequality. In the presence of correlation, however, the best one can achieve with this technique are broad 
intervals based on the Bonferroni inequalities. A second approach uses the fact that if the log relative risks are approximately normal, then the relative risks are approximately log normally distributed. Again, the joint distribution of log normal random variables is not straightforward. Consequently, this approach similarly becomes unworkable.

Instead, we follow Rothman (1976) and rely on a Taylor's series expansion. Specifically, we approximate the logarithm using:

$$
\begin{gathered}
\log y \doteq \log y_{0}+\frac{1}{y_{0}}\left(y-y_{0}\right) \text { so that } \operatorname{Var}(\log y) \doteq \frac{1}{y_{0}^{2}} \operatorname{Var}(y) \text {. Hence: } \\
\operatorname{Var}(y)=y_{0}^{2} \operatorname{Var}(\log y)
\end{gathered}
$$

The accuracy of the approximation improves for $y$ close to $y_{0}$, which we will achieve by taking $y$ to be a consistent estimate of $y_{0}$.

Collecting the terms required for the variance of the additive statistic, $\operatorname{Var}(A)$, we have:

$$
\begin{aligned}
\operatorname{Var}\left(R R_{A \& B}\right) & \doteq\left(R R_{A \& B}\right)^{2} \cdot\left[\frac{1}{h_{1}}+\frac{1}{h_{4}}+\frac{1}{k_{1}}+\frac{1}{k_{4}}\right] \\
\operatorname{Var}\left(R R_{A}\right) & \doteq\left(R R_{B}\right)^{2} \cdot\left[\frac{1}{h_{1}}+\frac{1}{h_{2}}+\frac{1}{k_{1}}+\frac{1}{k_{2}}\right] \\
\operatorname{Var}\left(R R_{B}\right) & \doteq\left(R R_{b}\right)^{2} \cdot\left[\frac{1}{h_{1}}+\frac{1}{h_{3}}+\frac{1}{k_{1}}+\frac{1}{k_{3}}\right]
\end{aligned}
$$

For the covariance terms we employ similar Taylor's expansions. Specifically let:

$$
\begin{aligned}
\log y & \doteq \log y_{0}+\frac{1}{y_{0}}\left(y-y_{0}\right) \text { and } \\
\log z & \doteq \log z_{0}+\frac{1}{z_{0}}\left(z-z_{0}\right) \text {. Then } \\
\operatorname{cov}(\log y, \log z) & \doteq \frac{1}{y_{0} z_{0}} \operatorname{cov}\left(y-y_{0}, z-z_{0}\right) \text { so that } \\
\operatorname{cov}(y, z) & \doteq\left(y_{0} z_{0}\right) \cdot \operatorname{cov}(\log y, \log z) . \text { Then: } \\
\operatorname{cov}\left(R R_{A}, R R_{B}\right) & =\left(R R_{A} \cdot R R_{B}\right) \cdot\left(\frac{1}{h_{1}}+\frac{1}{k_{1}}\right) \\
\operatorname{cov}\left(R R_{A \& B}, R R_{A}\right) & =\left(R R_{A \& B} \cdot R R_{A}\right) \cdot\left(\frac{1}{h_{1}}+\frac{1}{k_{1}}\right) \\
\operatorname{cov}\left(R R_{A \& B}, R R_{B}\right) & =\left(R R_{A \& B} \cdot R R_{B}\right) \cdot\left(\frac{1}{h_{1}}+\frac{1}{k_{1}}\right)
\end{aligned}
$$


Then we have:

$$
\begin{aligned}
\operatorname{Var}(A) \doteq & \left(R R_{A \& B}\right)^{2} \cdot\left[\frac{1}{h_{1}}+\frac{1}{h_{4}}+\frac{1}{k_{1}}+\frac{1}{k_{4}}\right] \\
& +\left(R R_{A}\right)^{2} \cdot\left[\frac{1}{h_{1}}+\frac{1}{h_{2}}+\frac{1}{k_{1}}+\frac{1}{k_{2}}\right] \\
& +\left(R R_{B}\right)^{2} \cdot\left[\frac{1}{h_{1}}+\frac{1}{h_{3}}+\frac{1}{k_{1}}+\frac{1}{k_{3}}\right] \\
& +2\left(R R_{A} \cdot R R_{b}\right) \cdot\left(\frac{1}{h_{1}}+\frac{1}{k_{1}}\right) \\
& -2\left(R R_{A \& B} \cdot R R_{A}\right) \cdot\left(\frac{1}{h_{1}}+\frac{1}{k_{1}}\right) \\
& -2\left(R R_{A \& B} \cdot R R_{B}\right) \cdot\left(\frac{1}{h_{1}}+\frac{1}{k_{1}}\right)
\end{aligned}
$$

A Wald test may be conducted using the ratio of $A$ to its standard error $\sqrt{\operatorname{Var}(A)}$. Asymptotically, this will be standard normal, given the limiting distribution of the joint multinomial probabilities for $\pi$ and $\theta$.

Since the Wald tests are valid only asymptotically we also consider a likelihood ratio approach.

\subsection{Maximum Likelihood}

The likelihood function for the case control study is $\prod_{i=1}^{4} \pi_{i}^{h_{i}} \theta_{i}^{k_{i}}$ and is maximized subject to the constraint $\sum \pi_{i}-1=\sum \theta_{i}-1=0$. The $\log$ likelihood function is

$$
F=\sum h_{i} \log \pi_{i}+\sum k_{i} \log \theta_{i}
$$

This is maximized subject to the constraints:

$$
\begin{gathered}
F_{1}=\sum \pi_{i}-1=0 \\
F_{2}=\sum \theta_{i}-1=0 \\
\text { and } F_{3}=\frac{\pi_{4} \theta_{1}}{\pi_{1} \theta_{4}}-\frac{\pi_{2} \theta_{1}}{\pi_{1} \theta_{2}}-\frac{\pi_{3} \theta_{1}}{\pi_{1} \theta_{3}}+1=0 \text { "additivity" or } \\
F_{4}=\log \left(\pi_{1} \theta_{2} \theta_{3} \pi_{4}\right)-\log \left(\theta_{1} \pi_{2} \pi_{3} \theta_{4}\right)=0 \quad \text { "multiplicativity" }
\end{gathered}
$$

Note that $F_{3}$ may be rewritten:

$$
\begin{aligned}
F_{3} & =\frac{\pi_{4}}{\theta_{4}}-\frac{\pi_{2}}{\theta_{2}}-\frac{\pi_{3}}{\theta_{3}}+\frac{\pi_{1}}{\theta_{1}} \\
& =\frac{\pi_{1}}{\theta_{1}}-\frac{\pi_{2}}{\theta_{2}}-\frac{\pi_{3}}{\theta_{3}}+\frac{\pi_{4}}{\theta_{4}}=0
\end{aligned}
$$




\subsubsection{Additive Constraint}

For the additive model, we maximize the Lagrangian

$$
F_{A}=F+\mu_{1} F_{1}+\mu_{2} F_{2}+\mu_{3} F_{3}
$$

where $\mu_{1}, \mu_{2}$, and $\mu_{3}$ are Lagrange multipliers. The first order conditions are:

$$
\begin{aligned}
& \frac{\partial F_{A}}{\partial \pi_{i}}=\frac{h_{i}}{\pi_{i}}+\mu_{1}+\frac{\delta_{i} \mu_{3}}{\theta_{i}}=0 \\
& \frac{\partial F_{A}}{\partial \theta_{i} i}=\frac{k_{i}}{\theta_{i}}+\mu_{2}-\frac{\delta_{i} \pi_{i} \mu_{3}}{\theta_{i}^{2}}=0 \quad \text { and } \\
& \frac{\partial F_{A}}{\partial \mu_{i}}=F_{i}=0 \quad i=1,2,3
\end{aligned}
$$

where $\delta_{1}=-\delta_{2}=-\delta_{3}=\delta_{4}=1$.

It follows that:

$$
\sum \pi_{i} \frac{\partial F_{A}}{\partial \pi_{i}}=h+\mu_{1}=0 \text { and } \sum \theta_{i} \frac{\partial F_{A}}{\partial \theta_{i}}=k+\mu_{2}=0
$$

Hence, $\hat{\mu}_{1}=-h$ and $\hat{\mu}_{2}=-k$ and the remaining conditions may be written:

$$
\begin{gathered}
\left(h_{i}-h \hat{\pi}_{i}\right) \hat{\theta}_{i}+\delta_{i} \hat{\pi}_{i} \hat{\mu}_{3}=0 \\
\left(k_{i}-k \hat{\theta}_{i}\right) \hat{\theta}_{i}-\delta_{i} \hat{\pi}_{i} \hat{\mu}_{3}=0 \\
\text { and } \sum \delta_{i} \frac{\hat{\pi}_{i}}{\hat{\theta}_{i}}=0
\end{gathered}
$$

Writing $x_{i}=\hat{\pi}_{i} / \hat{\theta}_{i}$ and solving the first order conditions implies:

$$
x_{i}=\frac{\left(\delta_{i} k_{i} h-k \hat{\mu}_{3}\right) \pm \sqrt{\left(\delta_{i} k_{i} h-k \hat{\mu}_{3}\right)^{2}-4 \delta_{i} h_{i} h k \hat{\mu}_{3}}}{2 \hat{\mu}_{3} h}
$$

Since $\sum_{i} \delta_{i} x_{i}=0$, it follows that:

$$
\begin{aligned}
0 & =\sum_{i}\left[\left(k_{i} h-k \hat{\mu}_{3} \delta_{i}\right) \pm \delta_{i} \sqrt{\left(\delta_{i} k_{i} h-k \hat{\mu}_{3}\right)^{2}-4 \delta_{i} h_{i} h k \hat{\mu}_{3}}\right] \\
& =k h+\sum_{i} \pm \delta_{i} \sqrt{\left(\delta_{i} k_{i} h-k \hat{\mu}_{3}\right)^{2}-4 \delta_{i} h_{i} h k \hat{\mu}_{3}}
\end{aligned}
$$

This equation in $\hat{\mu_{3}}$ may be solved for each of 16 possible sign combinations $(+$ or - for each of the four terms in the sum). 
Using $x_{i}=\pi_{i} / \theta_{i}$, the first two first order conditions may be written

$$
\left(k_{i}-k \theta_{i}\right)=\delta_{i} x_{i} \mu_{3} \quad \text { and } \quad\left(h_{i}-h \pi_{i}\right)=-\delta_{i} x_{i} \mu_{3}
$$

Hence, $\left(k_{i}-k \theta_{i}\right)=-\left(h_{i}-h \pi_{i}\right)$, which implies

$$
\begin{gathered}
k_{i}+h_{i}=k \theta_{i}+h \pi_{i}=\theta_{i}\left(k+h x_{i}\right) \quad \text { or } \\
\theta_{i}=\frac{k_{i}+h_{i}}{k+h x_{i}}
\end{gathered}
$$

Now substitute into the first order condition:

$$
\begin{aligned}
k_{i}-k\left[\frac{k_{i}+h_{i}}{k+h x_{i}}\right] & =\delta_{i} x_{i} \mu_{3} \text { or } \\
k_{i}\left(k+h x_{i}\right)-k\left(k_{i}+h_{i}\right) & =\left(k+h x_{i}\right) \delta_{i} x_{i} \mu_{3} \\
k_{i} k+k_{i} x_{i} h-k\left(k_{i}+h_{i}\right) & =k \delta_{i} x_{i} \mu_{3}+x_{i}^{2} h \delta_{i} \mu_{3} \\
-k h_{i} & =x_{i}\left(-k_{i} h+k \delta_{i} \mu_{3}\right)+x_{i}^{2} h \delta_{i} \mu_{3} \\
-k h_{i} & =x_{i}\left(k \delta_{i} \mu_{3}-k_{i} h\right)+x_{i}^{2} h \delta_{i} \mu_{3} \\
-k h_{i} \delta_{i} & =x_{i}\left(k \delta_{i}^{2} \mu_{3}-k_{i} h \delta_{i}\right)+x_{i}^{2} h \delta_{i}^{2} \mu_{3}
\end{aligned}
$$

Now use $\delta^{2}=1$ as $\delta=1$ or -1 . Then:

$$
\begin{gathered}
-k h_{i} \delta_{i}=x_{i}\left(k \mu_{i}-k_{i} h \delta_{i}\right)+x_{i}^{2}\left(h \mu_{3}\right) \quad \text { so that } \\
0=x_{i}^{2}\left(h \mu_{3}\right)+x_{i}\left(k \mu_{3}-k_{i} h \delta_{i}\right)+k h_{i} \delta_{i}
\end{gathered}
$$

The last equation establishes a bound on $x_{i}$ since the discriminant of the quadratic equation must be positive. The discriminant is:

$$
\begin{gathered}
\left(k \mu_{3}-k_{i} h \delta_{i}\right)^{2}-4\left(h \mu_{3}\right)\left(k h_{i} \delta_{i}\right) \geq 0 \\
k_{i}^{2} h^{2}-2 \delta_{i} k_{i} h k \mu_{3}+k^{2} \mu_{3}^{2}-2 \cdot 2 \delta_{i} h_{i} h k \mu_{3} \geq 0
\end{gathered}
$$




$$
\mu_{3}^{2}-2 \delta_{i}\left(\frac{h}{k}\right) \mu_{3}\left(k_{i}+2 h_{i}\right)+k_{i}^{2}\left(\frac{h}{k}\right)^{2} \geq 0
$$

Next, solving this quadratic at the point of equality to zero for $\mu_{3}$, we obtain:

$$
\begin{aligned}
\mu_{3 i}^{*} & =\frac{2 \delta_{i}\left(\frac{h}{k}\right)\left(k_{i}+2 h_{i}\right) \pm \sqrt{4 \delta_{i}^{2} \frac{h^{2}}{k^{2}}\left(k_{i}+2 h_{i}\right)^{2}-4 k_{i}^{2}\left(\frac{h}{k}\right)^{2}}}{2} \\
& =\delta_{i}\left(\frac{h}{k}\right)\left(k_{i}+2 h_{i}\right) \pm \sqrt{\frac{h^{2}}{k^{2}}\left(k_{i}+2 h_{i}\right)^{2}-k_{i}^{2}\left(\frac{h}{k}\right)^{2}} \\
& =\left(\frac{h}{k}\right)\left[\left(k_{i}+2 h_{i}\right) \delta_{i} \pm \sqrt{k_{i}^{2}+4 k_{i} h_{i}+4 h_{i}^{2}-k_{i}^{2}}\right] \\
& =\left(\frac{h}{k}\right)\left[\delta_{i}\left(k_{i}+2 h_{i}\right) \pm 2 \sqrt{h_{i}\left(h_{i}+k_{i}\right)}\right]
\end{aligned}
$$

Since the quadratic has a positive second derivative, the inequalities are $\mu_{3} \leq \min \mu_{3 i}^{*}$ and $\mu_{3} \geq \max \mu_{3 i}^{*}$.

Setting, $a_{i}=\left(\frac{h}{k}\right)\left[k_{i}+2 h_{i}-2 \sqrt{h_{i}^{2}+h_{i} k_{i}}\right]$, Gardner and Munford (1980) show that $-\min \left(a_{2}, a_{3}\right) \leq \hat{\mu}_{3} \leq \min \left(a_{1}, a_{4}\right)$.

Unfortunately, while these bounds bracket the true value of $\hat{\mu}_{3}$ they are not guaranteed to produce sign changes in the equation of interest. Therefore, an iterative solution is required to bracket each of the solutions for $\hat{\mu}_{3}$. We have found that $\hat{\mu}_{3}=0$ will always be a trivial solution to the equation above, and should be ignored.

Once $\hat{\mu}_{3}$ is found $\hat{\pi}_{i}$ and $\hat{\theta}_{i}$ are found from the first order conditions.

\subsubsection{Multiplicative Constraint}

For the multiplicative model, we maximize the Lagrangian:

$$
F_{M}=F+\lambda_{1} F_{1}+\lambda_{2} F_{2}+\lambda_{4} F_{4}
$$

$$
\text { with } \begin{aligned}
F & =\sum h_{i} \log \pi_{i}+\sum k_{i} \log \theta_{i} \text { and } \\
F_{1} & =\sum \pi_{i}-1 \\
F_{2} & =\sum \theta_{i}-1 \\
F_{4} & =\sum \delta_{i} \log \pi_{i}-\sum \delta_{i} \log \theta_{i}
\end{aligned}
$$


we have:

$$
\begin{gathered}
\frac{\partial F_{M}}{\partial \pi_{i}}=\frac{h_{i}}{\pi_{i}}+\lambda_{1}+\lambda_{4} \frac{\delta_{i}}{\pi_{i}}=0 \\
\frac{\partial F_{M}}{\partial \theta_{i}}=\frac{k_{i}}{\theta_{i}}+\lambda_{2}+\lambda_{4} \frac{-\left(\delta_{i}\right)}{\theta_{i}}=0 \text {. Then: } \\
\sum \pi_{i} \frac{\partial F_{M}}{\partial \pi_{i}}=\sum h_{i}+\lambda_{1}+\lambda_{4} \sum \delta_{i}=0 \Rightarrow \lambda_{1}=-h \\
\sum \theta_{i} \frac{\partial F_{M}}{\partial \theta_{i}}=\sum k_{i}+\lambda_{2}+\lambda_{4} \sum-\left(\delta_{i}\right)=0 \Rightarrow \lambda_{2}=-k \\
\frac{h_{i}}{\pi_{i}}-h+\frac{\lambda_{4} \delta_{i}}{\pi_{i}}=0 \quad \Rightarrow \quad h_{i}-h \pi_{i}+\lambda_{4} \delta_{i}=0 \\
h \pi_{i}=h_{i}+\lambda_{4} \delta_{i} \\
\pi_{i}=\frac{h_{i}+\delta_{i} \lambda_{4}}{h} \\
\frac{k_{i}}{\theta_{i}}+\lambda_{2}+\lambda_{4}\left(\frac{-\delta_{i}}{\theta_{i}}\right)=0 \Rightarrow \\
\frac{k_{i}}{\theta_{i}}-k+\lambda_{4}\left(\frac{-\delta_{i}}{\theta_{i}}\right)=0 \Rightarrow \\
k_{i}-k \theta_{i}+\lambda_{4}\left(-\delta_{i}\right)=0 \\
-k \theta_{i}=\lambda_{4} \delta_{i}-k_{i} \\
\lambda_{i}=\frac{\lambda_{4} \delta_{i}-k_{i}}{-k}=\frac{k_{i}-\lambda_{4} \delta_{i}}{h}
\end{gathered}
$$

Finally, substituting into the constraint implies:

$$
\left[\frac{h_{1}+\delta_{1} \lambda_{4}}{h}\right]\left[\frac{k_{2}-\delta_{2} \lambda_{4}}{k}\right]\left[\frac{k_{3}+\delta_{3} \lambda_{4}}{k}\right]\left[\frac{h_{4}+\delta_{4} \lambda_{4}}{h}\right]-\text { similar terms }=0
$$

which implies $\left(h_{1}+\lambda_{4}\right)\left(k_{2}+\lambda_{4}\right)\left(k_{3}+\lambda_{4}\right)\left(h_{4}+\lambda_{4}\right)-$ similar terms $=0$.

\subsubsection{Unconstrained Maximum Likelihood}

The log likelihood under the constraint of additivity or multiplicativity is $\sum h_{i} \log \hat{\pi}_{i}+$ $\sum k_{i} \log \hat{\theta}_{i}$. For the unconstrained case we maximize the Lagrangian

$$
L=\sum h_{i} \log \pi_{i}+\sum k_{i} \log \hat{\theta}_{i}+\psi_{1}\left[\sum \pi_{i}-1\right]+\psi_{2}\left[\sum \theta_{i}-1\right]
$$

The first order conditions are

$$
\begin{aligned}
& \frac{\partial L}{\partial \pi_{i}}=\frac{h_{i}}{\pi_{i}}+\psi_{1}=0 \quad \text { and } \quad \frac{\partial L}{\partial \psi_{1}}=\sum \pi_{i}-1=0 \\
& \frac{\partial L}{\partial \theta_{i}}=\frac{k_{i}}{\theta_{i}}+\psi_{2}=0 \quad \text { and } \quad \frac{\partial L}{\partial \psi_{2}}=\sum \theta_{i}-1=0
\end{aligned}
$$


These equations imply that $\hat{\pi}_{i}=h_{i} / h$ and $\hat{\theta}_{i}=k_{i} / k$ for the unconstrained maximum likelihood.

Hypothesis tests may be based on -2(log likelihood unconstrained - log likelihood constrained), which has a $\chi^{2}$ distribution with one degree of freedom.

While the additive and multiplicative models are non-nested, a comparison of the log likelihood values provides a basis for a non-nested hypothesis test.

\section{Bonferroni Intervals and Monte Carlo Simulations}

\subsection{Bonferroni Interval}

The additive statistic $A=R R_{A \& B}-R R_{A}-R R_{B}-1$ is composed of three random variables. A confidence interval for each component may be established using the variance of the log-odds ratio. Set at appropriate levels, these confidence intervals may be combined using basic results from probability theory. For a 95 percent confidence interval, chose a significance level such that one third of one half of 5 percent probability is in each tail of a normal distribution. Then:

$$
\operatorname{prob}[-2.39 \leq \mathrm{N}(0,1) \leq 2.39]=1-\frac{.05}{6}=0.98334
$$

Since $(\log \hat{R R}-\log R R) / \sigma \sim^{A} N(0,1)$ we have

$$
\operatorname{prob}[-2.39 \sigma \leq \log \hat{R R}-\log R R \leq 2.39 \sigma]=0.98334
$$

or

$$
\operatorname{prob}[-2.39 \sigma+\log \hat{R R} \leq \log R R \leq 2.39 \sigma+\log \hat{R R}]=0.98334
$$

so that

$$
\operatorname{prob}\left[\hat{R R e^{-2.39 \sigma}} \leq R R \leq \hat{R R e^{2.39 \sigma}}\right]=0.98334
$$

Similarly,

$$
\operatorname{prob}\left[\hat{R R_{A \& B}} e^{-2.39 \sigma_{R R_{A \& B}}} \leq R R \leq \hat{R R_{A \& B}} e^{2.39 \sigma_{R R_{A \& B}}}\right]=0.98334
$$

and so forth for $\hat{R R_{A}}$ and $\hat{R R_{B}}$. Similarly:

$$
\begin{gathered}
\operatorname{Prob}\left[C_{\text {low }}^{A \& B} \leq R R_{A \& B} \leq C_{\text {high }}^{A \& B}\right]=.98334 \\
\operatorname{Prob}\left[C_{\text {low }}^{A} \leq R R_{A} \leq C_{\text {high }}^{A}\right]=.98334 \\
\operatorname{Prob}\left[C_{\text {low }}^{B} \leq R R_{B} \leq C_{\text {high }}^{B}\right]=.98334
\end{gathered}
$$


Denoting the intervals within square brackets as $A, B$, and $C$, we have by the Bonferroni inequality:

$$
\operatorname{prob}[A \cap B \cap C] \geq 1-\left(P\left(A^{c}\right)+P\left(B^{c}\right)+P\left(C^{c}\right)\right)
$$

Then

$$
\begin{aligned}
\operatorname{prob}\left[C_{\text {low }}^{A \& B}\right. & \leq R R_{A \& B} \leq C_{\text {high }}^{A \& B} \cap \\
-C_{\text {high }}^{A} & \leq-R R_{A} \leq-C_{\text {low }}^{A} \cap \\
-C_{\text {high }}^{B} & \left.\leq-R R_{B} \leq-C_{\text {low }}^{B}\right] \geq 1-.05=.95
\end{aligned}
$$

so that

$$
\operatorname{prob}\left[C_{\text {low }}^{A \& B}-C_{\text {high }}^{A}-C_{\text {high }}^{B} \leq R R_{A \& B}-R R_{A}-R R_{B} \leq C_{\text {high }}^{A \& B}-C_{\text {low }}^{A}-C_{\text {low }}^{B}\right] \geq .95
$$

and

$$
\operatorname{prob}\left[C_{\text {low }}^{A \& B}-C_{\text {high }}^{A}-C_{\text {high }}^{B}-1 \leq A \leq C_{\text {high }}^{A \& B}-C_{\text {low }}^{A}-C_{\text {low }}^{B}-1\right] \geq .95
$$

As noted before, given the tendency of the intervals to be broad and imprecise, these intervals should be rejected in favor of Wald or Likelihood Ratio tests.

\subsection{Simulation Methods}

Consistent estimates of the $\pi_{j}$ and $\theta_{j}$ are formed using $h_{j} / h$ and $k_{j} / k$ respectively. A Monte Carlo technique draws a random multinomial deviate with marginal probabilities $\pi_{j}$ and $\theta_{j}$. Then, the empirical distribution of the statistics $M$ and $A$ are formed using repeated simulations. The empirical distributions establish confidence intervals centered around the realized value of the statistic. If these confidence intervals contain zero, then the hypothesis is not rejected.

\section{$4 \quad$ Synergy Indices}

\subsection{Rothman's $S$ Index}

Rothman (1976) considers the independently-acting agents $A$ and $B$ and a background effect $C$. $C$ is assumed to act independently of $A$ and $B$.

Let $P_{T}$ denote the probability that disease develops when both $A$ and $B$ are present in addition to the background $C . P_{A}$ is the probability that disease develops if $A$ were 
to act in isolation (without background). We define $P_{B}$ similarly. $P_{C}$ is the probability of getting disease from background only. Then

$$
\begin{aligned}
P_{T}= & P[A \cup B \cup C] \\
= & P[A]+P[B]+P[C]-P[A \cap B]-P[A \cap C]- \\
& P[B \cap C]+P[A \cap B \cap C]
\end{aligned}
$$

Now, under independence we have:

$$
P_{T}=P[A]+P[B]+P[C]-P[A] P[B]-P[A] P[C]-P[B] P[C]+P[A] P[B] P[C]
$$

Let $R_{A B}=P_{T}$ denote the combined risk.

Let $R_{A}=P[A \cup C]=P[A]+P[C]-P[A] P[C]$

Let $R_{B}=P[B \cup C]=P[B]+P[C]-P[B] P[C]$

Let $R_{0}=P[C]$

Then, under independence:

$$
\begin{aligned}
R_{A B}-R_{0} & =\left(R_{A}-R_{0}\right)+\left(R_{B}-R_{0}\right)-\frac{P_{A} P_{B}\left(1-P_{C}\right)\left(1-P_{C}\right)}{\left(1-P_{C}\right)} \\
& =\left(R_{A}-R_{0}\right)+\left(R_{B}-R_{0}\right)-\frac{\left(R_{A}-R_{0}\right)\left(R_{B}-R_{0}\right)}{\left(1-R_{0}\right)}
\end{aligned}
$$

Rothman's synergy index is defined as the ratio of the left-hand side of this equation to the right-hand side.

$$
S=\frac{\left(R_{A B}-R_{0}\right)}{\left(R_{A}-R_{0}\right)+\left(R_{B}-R_{0}\right)+\frac{\left(R_{A}-R_{0}\right)\left(R_{B}-R_{0}\right)}{\left(1-R_{0}\right)}}
$$

Under independence, the numerator and denominator will be equal and the synergy index will equal one. Ignoring the product terms in the denominator, which are likely to be small, Rothman's index becomes:

$$
S=\frac{\left(R_{A B}-R_{0}\right)}{\left(R_{A}-R_{0}\right)+\left(R_{B}-R_{0}\right)}=\frac{R R_{A B}-1}{R R_{A}+R R_{B}-2}
$$

where $R R_{A B}=R R_{A B} / R_{0}$ etc. When $S=1$, we obtain:

$$
\begin{aligned}
R R_{A B}-1 & =R R_{A}+R R_{B}-2 \text { or } \\
R R_{A B} & =R R_{A}+R R_{B}-1
\end{aligned}
$$


which we recognize as the additive hypothesis.

An alternative expression for Rothman's $S$ index is

$$
S=\frac{E R R_{A B}}{E R R_{A}+E R R_{B}}
$$

where $E R R_{A B}=R R_{A B}-1$ and $E R R_{A}=R R_{A}-1$ etc. Here, $E R R$ denotes excess relative risk.

\subsection{Attributable Proportion}

The attributable proportion is defined as the excess relative risk compared to the additive model divided by the combined relative risk. Formally,

$$
\begin{aligned}
A P & =\frac{E R R_{A B}-\left(E R R_{A}+E R R_{B}\right)}{\left(E R R_{A B}+1\right)} \\
& =\frac{\left(R_{A B} / R_{0}-1\right)-\left[\left(R_{A} / R_{0}\right)+\left(R_{B} / R_{0}-1\right)\right]}{\left[R_{A B} / R_{0}-1+1\right]} \\
& =\frac{R_{A B}-R_{0}-\left(R_{A}+R_{B}-2 R_{0}\right)}{R_{A B}} \\
& =\frac{R_{A B}-\left(R_{A}+R_{B}-R_{0}\right)}{R_{A B}} \\
& =\frac{R R_{A B}-\left(R R_{A}+R R_{B}-1\right)}{R R_{A B}}
\end{aligned}
$$

When the additive model is correct, $A P=0$.

Rothman's index $S$ and the attributable proportion $A P$ measure departure from additivity. They do not include the multiplicative hypothesis as a natural alternative. Therefore we consider an alternative which nests both hypotheses. 


\subsection{Additive-Multiplicative Measure}

Define

$$
\begin{aligned}
\gamma & =\frac{\left(R R_{A \& B}-1\right)-\left(R R_{A}-1\right)-\left(R R_{B}-1\right)}{\left(R R_{A}-1\right)\left(R R_{B}-1\right)} \\
& =\frac{R R_{A \& B}-R R_{A}-R R_{B}+1}{\left(R R_{A}-1\right)\left(R R_{B}-1\right)}
\end{aligned}
$$

Note that when $\gamma=0$ the additive hypothesis is true. When $\gamma=1$ we have:

$$
R R_{A \& B}=R R_{A}-R R_{B}+1=R R_{A} R R_{B}-R R_{A}-R R_{B}+1
$$

which implies: $R R_{A \& B}=R R_{A} \cdot R R_{B}$, i.e. the multiplicative hypothesis.

While difficult, a confidence interval may be derived by examining the distribution of $\log \gamma$. Note that

$$
\log \gamma=\log A-\left(\log \left(R R_{A}-1\right)+\log \left(R R_{B}-1\right)\right)
$$

where $A$ is the additive statistic. Then

$$
\begin{aligned}
\operatorname{Var}(\log \gamma)= & \operatorname{Var}(\log A)-\operatorname{Var}\left(\log \left(R R_{A}-1\right)\right)+\operatorname{Var}\left(\log \left(R R_{B}-1\right)\right) \\
& +2 \operatorname{cov}\left[\log \left(R R_{A}-1\right), \log \left(R R_{B}-1\right)\right] \\
& -2 \operatorname{cov}\left[\log A, \log \left(R R_{A}-1\right)\right] \\
& -2 \operatorname{cov}\left[\log A, \log \left(R R_{B}-1\right)\right]
\end{aligned}
$$

For case-control studies, we have previously derived these components. However, the utility of the expansion is questionable given that when the additive hypothesis is true, the log transformation is not defined.

\section{Cohort Studies}

Cohort studies derive standardized morbidity or mortality rates with reference to an external reference group. The standardized mortality rate (SMR) is also known as an observed to expected ratio because it is constructed by computing the expected number of outcomes (deaths) based on the external reference group's rates. Given the large samples from which they are typically based, the latter rates are assumed to be known without error.

The cohort method compares the death rates between groups for those exposed to contaminant $A$ (with or without exposure to $B$ ) and for those not exposed to contaminant $A$ (with or without exposure to $B$ ). For present purposes, contaminant $A$ will be smoking, while contaminant $B$ will be asbestos. Death rates are calculated and given in the following $2 \times 2$ table: 


\begin{tabular}{|l|c|c|}
\hline & non-smoking & smoking \\
\hline asbestos & $d_{A}^{N S}$ & $d_{A}^{S}$ \\
\hline non-asbestos & $d_{N A}^{N S}$ & $d_{N A}^{S}$ \\
\hline
\end{tabular}

The cohort method follows a group of individuals with some exposure to asbestos. Death rates are determined over time for this cohort. A sample of individuals from a non-asbestos exposed population is matched to the exposed population at the aggregate level (i.e., there is a similar number of individuals of each age group).

Before discussing the derivation of the death rates $d_{j}^{i}$, we note that cohort studies make each cell of the $2 \times 2$ table independent by design. This greatly simplifies the hypothesis testing and determination of confidence intervals. Relative risks are determined as follows:

$$
\begin{gathered}
R R_{A}=\text { relative risk of asbestos exposure }=d_{A}^{N S} / d_{N A}^{N S} \\
R R_{S}=\text { relative risk of smoking exposure }=d_{N A}^{S} / d_{N A}^{N S} \\
R R_{A S}=\text { the relative risk of combined exposure }=d_{A}^{S} / d_{N A}^{N S}
\end{gathered}
$$

The additive hypothesis is stated as:

$$
R R_{A S}-R R_{A}-R R_{S}+1=0
$$

or

or

$$
\frac{d_{A}^{S}}{d_{N A}^{N S}}=\frac{d_{A}^{N S}}{d_{N A}^{N S}}+\frac{d_{N A}^{S}}{d_{N A}^{N S}}-1
$$

$$
d_{A}^{S}=d_{A}^{N S}+d_{N A}^{S}-d_{N A}^{N S}
$$

or

$$
A^{*}=d_{A}^{N S}+d_{N A}^{S}-d_{A}^{S}-d_{N A}^{N S}
$$

Under additivity $A^{*}=0$.

The multiplicative hypothesis is stated as:

$$
R R_{A S}=R R_{A} \cdot R R_{S}=0
$$

or

$$
\frac{d_{A}^{S}}{d_{N A}^{N S}}-\frac{d_{A}^{N S}}{d_{N A}^{N S}} \cdot \frac{d_{N A}^{S}}{d_{N A}^{N S}}=0
$$

or

$$
d_{A}^{S} \cdot d_{N A}^{N S}-d_{A}^{N S} \cdot d_{N A}^{S}=0
$$

or

$$
\log d_{A}^{S}+\log d_{N A}^{N S}-\log d_{A}^{N S}-\log d_{N A}^{S}=0
$$


or

$$
M^{*}=\log d_{A}^{N S}+\log d_{N A}^{S}-\log d_{A}^{S}-\log d_{N A}^{N S}=0
$$

We note that the multiplicative statistic is similar to the additive statistic with the exception that it is stated as a sum of logarithms. This suggests that the two hypotheses may be nested using a Box-Cox transformation.

It is worth noting that (32) implies

$$
\frac{d_{A}^{N S}}{d_{A}^{S}}=\frac{d_{N A}^{N S}}{d_{N A}^{S}}
$$

which states that the columns in the table are proportional to one another. Similarly, the rows are in proportion if the multiplicative hypothesis is correct. These are common statements of independence and can be tested via Pearson Chi-squared statistics for such tables. Finally, given the relationship between contingency tables and the log-linear model, we should expect a direct test of the multiplicative hypothesis from the log-linear model.

Suppose $\log \left(P\left[Y_{1}, Y_{2}\right]\right)=\mu_{0}+\mu_{1} Y_{1}+\mu_{2} Y_{2}+\mu_{12} Y_{1} \cdot Y_{2} \quad$ Then

$$
\begin{aligned}
& \log (P(0,0))=\mu_{0} \\
& \log (P(0,1))=\mu_{0}+\mu_{2} \\
& \log (P(1,0))=\mu_{0}+\mu_{1} \\
& \log (P(1,1))=\mu_{0}+\mu_{1}+\mu_{2}+\mu_{12}
\end{aligned}
$$

If $P(0,0)$ is estimated by $d_{N A}^{N S}, P(1,0)$ by $d_{A}^{N S}, P(0,1)$ by $d_{N A}^{S}$, and $P(1,1)$ by $d_{A}^{S}$ (after suitable normalization), then the multiplicative hypothesis may be stated as:

$$
M^{*}=\left(\mu_{0}+u_{1}\right)+\left(\mu_{0}+\mu_{2}\right)-\left(\mu_{0}+\mu_{1}+\mu_{2}+\mu_{12}\right)-\left(\mu_{o}\right)=-\mu_{12}
$$

Then, $M^{*}=0$ (the multiplicative hypothesis) if and only if the interaction parameter $\mu_{2}=0$ in the log-linear model.

\subsection{Determination of Death Rates}

The death rate is defined as the number of deaths per 100,000 person years. This is typically measured by the number of deaths observed in the cohort divided by the number of person years multiplied by 100,000 . 
For example, suppose that a particular cohort has $N_{A}^{N S}$ individuals who are nonsmokers but who are exposed to asbestos. Suppose that these $N_{A}^{N S}$ individuals are followed for $Y_{A}^{N S}$ person years (on average $Y_{A}^{N S} / N_{A}^{N S}$ years per person). Suppose that $h_{A}^{N S}$ of these individuals die during the period of observation. Then

$$
d_{A}^{N S}=\left(\frac{h_{A}^{N S}}{N_{A}^{N S}}\right)\left(\frac{N_{A}^{N S}}{Y_{A}^{N S}}\right) \cdot 100,000
$$

The stochastic component in the expression is the binomially distributed random variable $h_{A}^{N S}$ that denotes the number of observed deaths in $N_{A}^{N S}$ trials. Let $P_{A}^{N S}$ denote the true but unobserved probability of dying. Then $\hat{P}_{A}^{N S}=h_{A}^{N S} / N_{A}^{N S}$ is a consistent estimate of $P_{A}^{N S}$.

$$
\text { Now } E\left(\hat{P}_{A}^{N S}\right)=P_{A}^{N S} \text { and } \operatorname{Var}\left(\hat{P}_{A}^{N S}\right)=\frac{P_{A}^{N S}\left(1-P_{A}^{N S}\right)}{N_{A}^{N S}} .
$$

Then

$$
\operatorname{Var}\left(d_{A}^{N S}\right)=\operatorname{Var}\left(\hat{P}_{A}^{N S}\right) \cdot\left[\frac{N_{A}^{N S}}{Y_{A}^{N S}}\right]^{2} \cdot 100,000^{2}
$$

When logarithmic transformations are employed we have

$$
\log d_{A}^{N S}=\log \hat{P}_{A}^{N S}+\log \left[\frac{N_{A}^{N S}}{Y_{A}^{N S}}\right]+\log (100,000) .
$$

Recall that a Taylor's series expansion shows that $\log \hat{P} \doteq \log P_{0}+\frac{1}{P_{0}}\left(\hat{P}-P_{0}\right)$ so that

$$
\operatorname{Var}(\log \hat{P})=\frac{1}{P_{0}^{2}} \frac{P_{0}\left(1-P_{0}\right)}{N}=\frac{\left(1-P_{0}\right)}{P_{0} N}
$$

Then

$$
\operatorname{Var}\left(\log \left(d_{A}^{N S}\right)\right) \doteq \frac{\left(1-\hat{P}_{A}^{N S}\right)}{\hat{P}_{A}^{N S} N_{A}^{N S}}
$$

Before proceeding with the formula for the variance of the $A^{*}$ and $M^{*}$ statistics, we note that replacing $P_{A}^{N S}$ by $\hat{P}_{A}^{N S}$ in the variance formula is valid asymptotically. Some researchers have noted that it may be more accurate in small samples to use a chi-square approximation.

To do this, we set $\chi^{2}=\frac{(\hat{P}-P)}{P(1-P) / N}$. Then we set the $\chi^{2}$ value to a critical level for the appropriate size test. Let $\chi_{r}^{2}$ be the critical value. Then

$$
\chi_{r}^{2}=\frac{(\hat{P}-P)^{2}}{P(1-P) / N}
$$


so that

$$
\begin{aligned}
\hat{P}-2 \hat{P} P+P^{2} & =\chi_{r}^{2} P(1-P) / N \\
& =\frac{P}{N} \chi_{r}^{2}-\frac{P^{2}}{N} \chi_{r}^{2}
\end{aligned}
$$

Then

$$
P^{2}\left(\frac{\chi_{r}^{2}}{N}+1\right)+P\left(\frac{-\chi_{r}^{2}}{N}-2 \hat{P}\right)+\hat{P}^{2}=0
$$

is a quadratic equation that may be solved for $P$. A confidence bound is derived using the two solutions of the quadratic equation.

\subsection{Variance of the Additive and Multiplicative Statistics}

Next, we derive the variance of the additive and multiplicative statistics for cohort studies. Recall that

$$
A^{*}=\left(d_{A}^{N S}-d_{A}^{S}\right)-\left(d_{N A}^{N S}-d_{N A}^{S}\right)
$$

For the non-asbestos exposed cohort, the rates $d_{N A}^{N S}$ and $d_{N A}^{S}$ are determined from large samples and are considered non-stochastic. Therefore the variance is determined from the components $d_{A}^{N S}$ and $d_{A}^{S}$, which are stochastic but independent. In this case,

$$
\begin{aligned}
\operatorname{Var}\left(A^{*}\right)= & \operatorname{Var}\left(d_{A}^{N S}\right)+\operatorname{Var}\left(d_{A}^{S}\right) \\
= & {\left[\frac{\hat{P}_{A}^{N S}\left(1-\hat{P}_{A}^{N S}\right)}{N_{A}^{N S}}\right]\left(\frac{N_{A}^{N S}}{Y_{A}^{N S}}\right)^{2} \cdot(100,000)^{2}+} \\
& {\left[\frac{\hat{P}_{A}^{S}\left(1-\hat{P}_{A}^{S}\right)}{N_{A}^{S}}\right]\left(\frac{N_{A}^{S}}{Y_{A}^{S}}\right)^{2} \cdot(100,000)^{2} }
\end{aligned}
$$

For the multiplicative statistic,

$$
M^{*}=\left[\left(\log d_{A}^{N S}\right)-\left(\log d_{A}^{S}\right)\right]-\left[\left(\log d_{N A}^{N S}\right)-\left(\log d_{N A}^{S}\right)\right]
$$

so that

$$
\operatorname{Var}\left(M^{*}\right)=\frac{\left(1-\hat{P}_{A}^{N S}\right)}{\hat{P}_{A}^{N S} N_{A}^{N S}}+\frac{\left(1-\hat{P}_{A}^{S}\right)}{\hat{P}_{A}^{S} N_{A}^{S}}
$$


These variances are used to calculate standard errors, confidence intervals, and Wald tests for the additive and multiplicative hypotheses.

For instance, $M^{*} / \sqrt{\operatorname{Var}\left(M^{*}\right)}$ is asymptotically standard normally distributed under the null hypothesis that $M^{*}=0$.

\subsection{Variance of the Synergy Index}

To derive the variance of $S, \operatorname{Var}(S)$ we first find $\log S$.

$$
\log S=\log \left(R_{A B}-R_{0}\right)-\log \left[\left(R_{A}-R_{0}\right)+\left(R_{B}-R_{0}\right)\right]
$$

For cohort studies, we have:

$$
\begin{aligned}
\log S & =\log \left(R R_{A B}-1\right)-\log \left[\left(R R_{A}-1\right)+\left(R R_{B}-1\right)\right] \\
& =\log \left(\frac{d_{S}^{A}}{d_{N A}^{N S}}-1\right)-\log \left[\left(\frac{d_{A}^{N S}}{d_{N A}^{N S}}-1\right)+\left(\frac{d_{N A}^{S}}{d_{N A}^{N S}}-1\right)\right] \\
& =\log \left(d_{A}^{S}-d_{N A}^{N S}\right)-\log \left(d_{A}^{N S}+d_{N A}^{S}-2 d_{N A}^{N S}\right) \\
\operatorname{Var}(\log S) & =\frac{\operatorname{Var}\left(d_{A}^{S}\right)}{\left(d_{A}^{S}-d_{N A}^{N S}\right)^{2}}+\frac{\operatorname{Var}\left(d_{A}^{N S}\right)}{\left(d_{A}^{N S}+d_{N A}^{S}-2 d_{N A}^{N S}\right)^{2}}
\end{aligned}
$$

where we have used the fact that $\operatorname{Var}\left(d_{N A}^{S}\right)=\operatorname{Var}\left(d_{N A}^{N S}\right)=0$ in cohort studies since these variables are assumed to be non-stochastic.

For case-control studies, we have:

$$
\operatorname{Var}(\log S)=\frac{\operatorname{Var}\left(R R_{A B}\right)}{\left(R R_{A B}-1\right)^{2}}+\frac{\operatorname{Var}\left(R R_{A}\right)+\operatorname{Var}\left(R R_{B}\right)+2 \operatorname{cov}\left(R R_{A}, R R_{B}\right)}{\left(R R_{A}+R R_{B}-2\right)^{2}}
$$

where the relevant components were derived above in the case-control section.

\section{Conclusion}

Case-control, cohort, and prevalence studies provide varying types of information to determine relative risks and attendant confidence levels. We have considered several methods for testing additivity and multiplicativity hypotheses using Wald and likelihood ratio techniques. In these cases, we have relied on asymptotic expectation for which the 
small sample populations are unknown. Our empirical results are reported in a companion paper and, generally, we find agreement in our conclusions regarding the additivity or multiplicativity hypothesis whether the analysis is conducted using Wald or likelihood ratio methods. 


\section{References}

[1] Gardner, M. J., Munford, A. G., (1980). "The combined effect of two factors on disease in a case-control study." Applied Statistics, 29: No. 3, 276-281.

[2] Rao, C. R., (1973). Linear Statistical Inference and its Applications, Wiley, New York.

[3] Rothman, K. J., (1976). "The estimation of synergy or antagonism." American Journal of Epidemiology, 103: No. 6, 506-511.

[4] Woolf, B. (1955). "On estimating the relation between blood group and disease." Annals of Human Genetics, 19: 251-253. 